Original Research Article

\title{
Research Progress of Porcine Reproductive and Respiratory Syndrome
}

\author{
Xueqi liao,Xianming Fan,Fengxue Qu \\ Animal Science and Technology College, Luohe Agricultural University, Henan, China
}

\begin{abstract}
Porcine Reproductive and Respiratory Syndrome (PRRS) is caused by Porcine Reproductive and Respiratory Syndrome Virus (PRRSV), which is characterized by sow reproductive disorder and piglet respiratory syndrome. The complex pathogenesis of PPRS includes antibody-dependent enhancement, immune suppression and persistent infection in the body and viral genetic diversity. In order to have a better understanding of the occurrence and prevalence of porcine reproductive and respiratory syndrome in China. The review of PRRS etiology, epidemiology, pathogenesis, genetic variation, diagnosis and control technology and other aspects of the progress were carried out.

KEYWORDS: porcine reproductive and respiratory syndrome; etiology; epidemiology; diagnosis; control technique
\end{abstract}

\section{Introduction:}

Porcine Reproductive and Respiratory Syndrome (PRRS) is also known as 'pig ear disease', which caused pig respiratory tract infection, piglet death, sow reproductive disorders and other clinical manifestations. 1987, the United States first reported the occurrence of the disease [1], Guo Baocai et al. [2] for the first time he successfully isolated PRRS virus from the domestic suspected PRRS infected pigs. The disease is caused by porcine reproductive and respiratory syndrome virus (PRRSV), PRRSV is a capsule of single stranded positive strand RNA virus, with a high degree of variability [3], derived from different regions PRRSV, the nucleotide sequence of the strains was significantly different. According to these differences, PRRSV could be divided into two genotypes, American and European, and the nucleotide sequence homology between the two genotypes was only 55\% - 80\% [4]. This article described the PRRS etiology, diagnosis and control techniques.

\section{PRRS etiology}

\subsection{1 General characteristics}

PRRSV belongs to the genus Nidovirus, the pathogen of the arteritis virus, the aristitis virus, it was a capsule of single stranded positive strand RNA virus. In addition, same as the equine arteritis virus (EAV), mouse lactate dehydrogenase virus (LDV) and monkey hemorrhagic fever virus (SHFV) and so on. PRRSV, LDV, EAV and SHFV had some similarities in morphology, cell tropism, replication system and protein structure. However, the morphological structure, replication characteristics, cell tropism, epidemiological transmission and the characteristics of the disease caused by the two genotypes of PRRSV were the same. The virus is spherical, 20-sided symmetrical, relatively smooth surface, cubic nucleocapsid, nucleolus shell around the lipid bilayer membrane, the diameter between $45 \mathrm{~nm}-65 \mathrm{~nm}$, containing $20 \mathrm{~nm}-35 \mathrm{~nm}$ core [ 5]. PRRSV strain can grow on porcine primary lung macrophages (PAM) and CL2621, Marc-145 and other passaged cell lines, and produced plaque phenomenon, which is characterized by the increased initial refraction of infected cells, Agglomeration and through the extend of time the cell shrinkage, and finally dissolve off.

\subsection{Molecular biological characteristics}

PRRSV genome length is about $15 \mathrm{~kb}$, merogenetic and consist 8 ORFs such as ORFs, ORF2, ORF3, ORF4, 0FF5, 0RF6 and ORF7, each ORF (from ORFl ORF7) and the adjacent ORF parts overlap, in their 5 ', 3' there is a non-

Copyright (C) 2017 -. This is an Open Access article distributed under the terms of the Creative Commons Attribution-NonCommercial 4.0 International License (http://creativecommons.org/licenses/by-nc/4.0/), permitting all non-commercial use, distribution, and reproduction in any medium, provided the original work is properly cited. 
coding area respectively. There is a 'hat' structure in front of the 5 'noncoding region, and polyadenylate (PolyA) tail after the 3' untranslated region. ORFla and ORFlb constitute $80 \%$ of the viral genome, encoding the viral replicase RNA polymerase. ORF2 - ORF7 encodes a viral structural protein in which ORF2 ORF5 encodes a viral glycosylated protein, ORF6 encodes a membrane matrix protein (M protein), and ORF7 encodes a nucleocapsid protein (N protein). ORF1 encodes viral replicase, after the process of encoding the oligosaccharides by ORF1a, it forms 6 types of nonstructural proteins such as (Nspla, Nsplp, Nsp2 - Nsp5), among the rest Nsp2 is very different from the American and European strains, its amino acid homology is only $32 \%$. ORFlb encodes oligosaccharides by about 463 amino acids, cleaved by ORFla-encoded proteases and forms four proteins, RdRp, CP2, CP3 and CP4 [6]; GP2 is encoded by oRF2 glycoprotein, with molecular mass of $29 \mathrm{ku}-30 \mathrm{ku}$. Both genotypes of PRRSV GP2 contained two distinct hydrophobic peaks and two derived $\mathrm{N}$ glycosylation sites [7]. GP3 is encoded by ORF3 and has a molecular weight of $27 \mathrm{ku}-29 \mathrm{ku}$. It is one of the most conserved proteins among PRRSV strains. The homology of American and European strain amino acid is 54\% - 60\%, and most of the mutation occurs at the N-terminus [8]; GP4 is a glycosylated envelope protein encoded by ORF4 with a molecular weight of $19 \mathrm{ku}-20 \mathrm{ku}$, which contains four glycosylation sites. For instance, the N and C-terminal region is a highly hydrophobic region [9]; GP5 is a glycosylated envelope protein encoded by ORF5, also known as E protein, molecular weight of about $22.4 \mathrm{ku}$, American and European isolates of the GP5 are composed of 200 and 201 amino acids, there are six antigenic determinants that can induce the body to produce specific neutralizing antibody [10]; M protein is ORF6 encoded by the membrane matrix protein with molecular weight of $18-19 \mathrm{ku}$. The amino acid sequence of $\mathrm{M}$ protein from European and American strains was the most conserved, with 173 and 174 amino acids respectively, and the homology was 78\% - 81\%. Besides, for N protein that was encoded by ORF7 Nucleocapsid protein, with molecular weight of $14 \mathrm{ku}-15 \mathrm{ku}$. It is the smallest major structural protein in PRRSV, PRRSV American and European type virus strains N-type protein with the number of amino acids of 123 and 128 respectively. Moreover, the N-terminal and C-terminal of the European strain N protein have additional two more amino acid extension regions compare to the American strain which are STAPM and SQGAS [ 12].

\subsection{Genetic variation and antigen diversity}

There is a strong variability in the PRRSV strain. The antigenicity and virulence of different strains are different. Although there are serological crosses in the American and European isolates, most of them have significant antigenic differences. The antigen diversity and genetic variation of the PRRSV strain are not only present between the two serotypes of North America and Europe, but also in the same serotypes of different strains, and even the vaccine has been mutated over the long term through the pig. The existing sequence analysis showed that the variation among European strains was significantly less than American strains, also ORF3 and ORF5 were the two least conserved study scope in the coding region of structural proteins. The American and European strains M-protein is most conserved with homology of amino acid of $78-81 \%$. The difference of amino acid sequence of GP5 protein was the highest, and the homology was only $51-55 \%$. In the epidemiology of PRRSV I rhyme, in order to identify PRRSV vaccine strains and American type PRRSV wild-type strains, Cheon DS et al. [13] used RFLP analysis to compare the PRRSV attenuated vaccine strain with its ancestral strain VR-2332 and other 22 PRRSV isolates the nucleotide sequence of ORF5 and the amino acid coding sequence of ORF5. It showed that the amino acid at position 151 of ORF5 encoded amino acid changed from arginine of wild strain to glycine of vaccine strain, and it was thought that the mutation of amino acid at this point was vaccine strains are specific and can be used for the identification of attenuated PRRS vaccine strains. But base mutations in ORF5 may be more than that, and the accumulation of these mutations has led to changes in the virulence of PRRSV [14]. In the process of proliferation of the vaccine, the genome of the vaccine has a selective mutation to the virulent strain, especially the selective mutation ability of ORF5 is the strongest.

\section{4. pathogenesis}

The way PRRSV infects cells first began with combined with receptors on porcine alveolar macrophages and then enters the cells through endocytosis. As the cells collapse, it enters into the blood circulation and lymph circulation, the result can lead to the formation of viremia and Systemic lymph node infection [15]. It has been reported that the most significant changes caused by PRRSV are severe damage to alveolar macrophages, causing a large amount of damage to the cells, as well as circulatory lymphocytes and chorionic cleavage system destruction, thereby inhibiting the immune system, so that the pig prone to other diseases infection. Studies have shown that many of the symptoms and lesions are closely related to microcirculation disorders, the central nervous system microcirculation disorder caused the increased of vascular permeability, the blood part of the liquid and tangible components of varying degrees of exudation, resulting in perivascular edema, bleeding or tube also the formation of the brain and spinal cord parenchymal focal bleeding. These changes and clinical observation of anorexia, depression, lethargy or even temperature rise or death is closely related. As for the blue ear and limbs hard, in addition to local microcirculation disorders, it may also be associated with pulmonary circulation disorders, with macrophage hyperplasia of alveolar septum thickening and tracheal, bronchial lesions are directly related to the virus. 


\subsection{Molecular epidemiology}

The GP5 gene had the highest variation among the genomes of PRRSV, and the nucleotide homology of GP5 gene was $89 \%-94 \%$ in American type PRRSV strain, and the homology of GP5 gene for European PRRSV strains was $87.1 \%$ to $99.5 \%$ [16]. GP5 gene mutation analysis has become a hotspot in PRRSV molecular epidemiology [17]. North American and Asian are commonly prevalent with American PRRSV strains. Where European PRSSV strains are commonly prevalent in Europe. But with economic globalization, the widespread use of PRRSV attenuated vaccines made PRRSV popular and there have been some new situations. Madsen KG et al. [18] used RT-PCR to detect the disease tissue of a pig farm in Denmark. By analyzing the nucleotide sequence ORF2 '- ORF7, it was found that the homology of the sequence with VR-2332 Between $99.2 \%$ and $99.5 \%$, while the VR-2332 strain is the ancestral strain of the attenuated vaccine used in Denmark and it may be associated with the spread and virulence of the American attenuated vaccine strain. In addition, the European and American type PRRSV coexisted in Denmark. Thanawonguwech R et al. [19] used the mnRT-PCR method to amplify the PRRSV ORF5 gene in the pig tissue of Thailand, and found that among the 137 samples. The positive rate of PRRSV was $66.42 \%$ and the positive rate of PRRSV in the Americas was 33.58\%. The Thailand European type PRRSV isolates nucleotide sequence of ORF5 gene with LV homology of $87-97.5 \%$. The American PRRSV isolates the sequence of nucleotides with $89 \%-90 \%$ homologous to that Canadian PRRSV (IAF-EXP91) isolate. However, the European type of PRRSV attenuated vaccine was not used in Thailand before, this might be the cause of Introduction breeding that brought the European type PRRSV into Thailand. 2006 China Zoo Disease Prevention center isolates the PRRSV mutant from the Jiangxi pig farms, In the serum of the American strain and the European strain PRRSV, the first use of RNA PRRSV molecular epidemiology research on the effective control of PRRS is of great significance.

\section{PRRS diagnostic techniques}

\subsection{Clinical symptoms and pathological changes}

Sows repeatedly show body temperature rise, loss of appetite, drowsiness, difficulty breathing, late miscarriage, premature birth, dead fetus, fetal mummification, individual pig ears, ventral and vulvar skin showing purple or purple plaque. There are also difficulties in lactation, childbirth difficulties, secondary cystitis, repeated estrus, with infertility and other symptoms. In addition to the boar fever, anorexia and cough and other symptoms, there are also temporary semen quality decline, decreased sperm motility, abnormal sperm increased, 13 months after the onset of symptoms returned to normal. Growing fattening pigs after the onset of dyspnea, cough, ears, abdomen and genital skin thick, it is also known as 'blue ear disease.' Just weaned piglets often secondary to some viral or bacterial disease and causes mortality increased, the main lesion is commonly the pulmonary diffuse interstitial pneumonia. Piglets grow dysplasia, often die within the first week of birth, difficulty breathing and sometimes visible conjunctivitis and edema of the eye. Lactation piglets body temperature rises, diarrhea, eyelid swelling, conjunctivitis, limb outreach, body dehydration, physical weakness, difficulty breathing, bleeding symptoms, the skin has a lot of plaques, and some even with joint disease, sepsis and so on. PRRSV caused by pathological changes, the most common is interstitial pneumonia, histological examination can be seen alveolar wall thickening, mononuclear cells and macrophages infiltration, alveolar epithelial cell degeneration, alveolar cavity protein fragments, a lot of Alveolar macrophages are destroyed. Brain main lesions is in the brain stem, showing subacute mononuclear encephalitis and perivascular inflammation, around in the small blood vessels there are presence of macrophages, lymphocytes, plasma cells and nuclear debris and other components that formed the 'vascular sets.' Sometimes it happened in the heart, kidney, lymph nodes, liver or other organs also occur in varying degrees of tissue lesions, such as myocardial atrophy, renal cortical point of bleeding and so on.

\section{2. virus isolation and RT-PCR detection}

Virus isolation (VI) is the most reliable way to diagnose the disease. When the virus is separated, it is best to use the lungs, heart, brain, kidney, liver, spleen and other organs of stillbirth or live piglets. The serum of the aborted fetus in late pregnancy is also used to separate PRRSV samples. It is difficult to isolate the virus from the mummy Pig. semen can't be used for the separation of PRRSV because semen has greater toxicity to cells [20]. It was found that different strains were suitable for proliferation on different cells. Most PRRSV strains were adapted to proliferate in PAM, but the cost of PAM was high and difficult. Egli C et al. Reported the use of quantitative TaqMan RT-PCR to detect PRRSV. Compared with other methods, it is found that there is very good performance in time saving, ease of use, sensitivity and specificity, Besides, it also can reduce the risk of cross-contamination. Hoeller K et al. Developed a one-step single-tube real-time RT-PCR technique to detect the extraction of an exogenous mRNA into each sample. RNA was isolated using a thiocyanate method such as phenyl guanidine Primers and probes, as well as all isolates of Europe and the Americas are labeled in primers. This method can provide absolute and relative number of virus in the serum and serologically monitor the swine in the farm. 


\subsection{Serological diagnosis}

The serological diagnostic method is the most widely used diagnostic method. For the serological diagnosis of PRRSV, four PRRSV antibody detection methods have been established at China and abroad. Namely, immunoperoxidase monolayer test (IPMA), indirect fluorescent antibody test (IFA), serum neutralization test (SN) and enzyme Immunosorbent assay (ELISA). IPMA is a method widely used in European countries to diagnose the disease. The sensitivity and specificity are good, and can be used for virus identification and serum antibody detection of PRRSV. This method has been widely used in PRRSV infection in Europe and America. IFA and IPMA have the same specificity and sensitivity, and is widely used in North America. SN detection PRRS antibody specificity is good, but generally in the infection after 4 weeks to 6 weeks before the emergence of neutralizing antibodies, so SN is not suitable for early diagnosis of PRRS. ELISA is fast, economical and sensitive, suitable for the detection of a large number of samples, the results of the decision without subjective factors, can be semi-automatic (machine wash plate) and automatically display the results. Significantly better than IPMA and IFA, has been used as PRRS immune monitoring and the routine of diagnosis.

\section{PRRS control technology}

\section{1. immunization prevention}

\subsubsection{Inactivated Vaccine PRRSV}

Inactivated vaccines are safe, and there is no risk of spreading the virus and causing a new source of PRRS, which is convenient for storage and transportation, and is not sensitive to interference from maternal antibodies. It is the vaccine that is preferred for the prevention and control of PRRS. Du-ran P et al reported that the use of Spanish strains developed into an oil adjuvant inactivated vaccine for the protection of sows. Basal immunization was performed twice in intramuscular injection at intervals of 21 days. During the lactation one time injection was needed to strengthen the immune system of the sows. The challenge test for pregnant sows showed that the immune protection rate was $70 \%$, and it was proved safe and effective by field test of 500,000 copies.

\subsection{2 attenuated vaccine}

The attenuated vaccine is suitable for pigs from 3 weeks to 18 weeks of age and can be immunized within 7 days after vaccination and the effective protection will be provided at least within 16 weeks. Benfield D A determine the weight and weight gain of prenatal and postnatal piglets show that pregnant sow vaccination of attenuated vaccine provided certain degree of protection on the subsequent virulence (NADC28) attack. It also confirms the completeness of immune protection of homologous strains, whereas intrauterine vaccination of NADC28 strains provides only limited protective immunity against the isolates of Lelystad strain. Compared with inactivated seedlings. The attenuated vaccine, while providing immunization protection, continues to spread the vaccine virus, allowing the virus to circulate in the pig farm and can't detect immunized pigs and naturally infected pigs by serological methods. Nielsen $\mathrm{J}$ et al. vaccine from the fetus, stillbirth and death piglets isolated from the infection of late pregnant sows, proved that the vaccine source can continue to exist in the herd, and cause disease. In short, live vaccines should be used with caution, at least in the promotion of the need for a large number of comprehensive laboratory research to accumulate enough data.

\subsection{3 nucleic acid vaccine}

It has been reported that anti-PRRSV neutralizing antibodies can be detected after vaccination of GP5 proteins expressed with baculovirus; GP3 immunization with baculovirus expression can even induce a higher protective effect than GP5 protein immunization. And found that through the recombinant of GP3-GP5 protein immunization can get the strongest protective effect. Plasmid (BCG) was used to express GP5- deficient recombinant (rBCGGP5) and M protein recombinant ( $\mathrm{rBCGM})$. After 30 days of inoculation, the recombinant BCG vaccine produced a specific humoral immune response. After 60 days of inoculation, Detection of neutralizing antibodies, also detected anti-BCG antigen IFN-Arab reaction, established a partial protection of PRRSV.

\subsection{2 Comprehensive prevention and control}

Currently there is no special effective control method for this disease, which is caused by a variety of reasons: PRRSV is prone to mutation, thereby it escapes the immune response; a pig farm may also infect one or several strains, PRRSV immunity Complex and specific, sometimes viremia and circulating antibodies exist at the same time, and PRRSV itself destroyed the immune cells, which is easy to form a persistent infection in the pig; pigs infected with 
PRRSV are very easy secondary to other low virulence pathogens Mixed infection, the formation of a variety of disease syndrome, such as respiratory disease syndrome (PRDC); PRRSV transmission capacity is very strong, sometimes 170177. Even after taking very stringent biosecurity measures or means of production.

In view of the characteristics of PRRS, the following aspects of comprehensive control measures can be taken: pig farm or scale pig farms should strictly implement the disinfection system, limit the visiting field, the necessary admission staff should change clothes, shoes, and utensils should be disinfected. The general efficacy of classical swine fever virus can be used for the disinfection of the disease, such as peracetic acid, a variety of organic acids. breeding pigs to implement 'all into the whole' system, each batch before and after, Pig farm must be strictly clean and disinfected. Lactation pig early weaning, maternal isolation and breeding, different age pig herd isolation and they were kept in different field areas. In this case, it can reduce the opportunity of virus being transmit from old pigs to piglets to help eliminate the persistent infection of pigs; PRRSV removal program, The PRRSV antibodies and antigens in the serum were monitored by ELISA and RT-PCR. The monitoring system of whole breeding group was established, and cleared out the infected pigs, so as to prevent the transmission of PRRSV.

\section{References:}

1. Keffaber K K. Reproductive failure of unknown etiology [J]. Death by apoptosis [J '1.J Virol, 1997.71 (12), 9170-9179.Swine Pract, 1989, 18 (31) 11-9.

2. Guo Baocheng, Chen Zhangshui, Liu Wenxing, et al. Study on the isolation of porcine reproductive and respiratory syndrome virus from suspected fetal PRRS abortion [J]. Chinese livestock and poultry infectious diseases. 1996, 22 2).

3. HollandJ, Spindler K, Horodyski F, et a1. Rpid evolution of vi-ral genornes [J]. Science, 1982, 21511577-1585.

4. Meulenberg J J, Petersen den Beaten A, de Kluyver E, et al. Molecular characterization of Lelystad, irus [J]. Vet Microbiol, 1997, 31 (55): 197-202.

5. Wensvoort G. Lelystad virus, the cause of porcine epidemic abortion and respiration syndrome 1 a view of mystery swine disease research at Lelystad [J]. Vet Microbiol, 1992, 33I 185192.

6. Allende R. Mutation in the genome of Porcine reproductive and respiratory syndrome virus responsible for the attenuation phenotype [J]. Arch Virol, 2000, 14511149-1161.

7. MengX J. Paul P S, Halbur P G, et al. Sequence comparison of open reading frame 2 to 5 of 10 W and high virulence United States isolates of porcine reproductive and respiratory syndrome virus [J ]. J Gen Vir 01.1995.76: 3181-3188.

8. Murtaugh MP, Elam MR, Kakaeh LT. et al. Comparison of the structural protein coding sequences of the VR-2332 and Lelystad virus strains of the PRRS virus [J]. Arch Vir01.2005. 140 I 1451-1460.

9. Mardassi H, Mounir S, Gagnon C A, et al. Molecular analysis of the oRF3-7 of porcine reproductive and respiratory syndrome virus. Quebec reference strain. Arch Virol, 2005, 140i1405-1418.

10. 103 Pirzadeh B, Gagnon C A. Dea S. Genomic and antigenic variations of porcine reproductive and respiratory syndrome major envelope GP5 glycoprotein [J]. Can J Vet Res, 1998.

11. Meng J. Heterogeneity porcine reproductive respiratory syndrome virus implications for current vaccine efficacy and future vaccine development Vet Mierobiol. 2000, 74 1309-329.

12. Dea S, Gagron C A, Mardaasi H, et al. Antigenic variability among North American and European strains of procine reproductive and respiratory syndrome as defined by monoclonal antibodies to the matrix protein. J Clin Microbiol, 2006, 34 (6) 11488-1493.

13. Cheon D S, Chae C. Restriction fragment length polymorphism analysis of open reading frame 5 gene of porcine reproductive and respiratory syndrome virus isolates in Korea [J]. Areh Virol, 2000.1451 1481-1488.

14. Shen S. Determination of the complete nucleotide sequence of vaccine strain of porcine reproductive and respiratory syndrome virus and identification of the Nsp2 gene with a unique insertion [J]. Arch Virol, 2000.145 1871-883.

15. Sur J H. Doster A R. Christian J S, et al. Porcine reproductive and respiratory syndrome virus replicates in testicular germ cells. Alters spermatogenesis And induces germ cell.

16. Forsberg R, Storgaard T, Nielsen H S, et al. The genetic diversity of European type PRRSV is similar to that of the North American type but is geographically skewed within Europe [J]. Virology. 2002, 293 S-47.

17. Ansari I H, Kwon B J, Osorio F A, et al. Influence of N-linked glycosylation of porcine reproductive and respiratory syndrome virus GP5 on virus infectivity. Antigenicity, and ability to belief neutralizing antibodies [J]. J Virol, 2006, 99 13994-4004

18. Analysis of porcine reproductive and respiration syndrome virus of the American type collected from Danish swine herds [J]. Arch Virol. 1998.143 (9) I

19. Thanawongnuwech R. Amonsin A. Tatsanakit A, et al. Genetics and geographical variations of porcine reproductive and respiratory syndrome virus (PRRSV) in Thailand [J]. Vet MicrobioI. 2004.10

20. Mengeling W L, Lager K M. A brief review of procedures and potential problems associated with the diagnosis of porcine reproductive and respiratory syndrome [J]. Vet Res. 2000. 